\title{
Measurements of middle ear functioning- traditional (acoustic impedance and acoustic admittance) and modern (middle ear reflectance and middle ear absorbance): a review
}

\author{
Udit Saxena $^{1 *}$, S. B. Rathna Kumar ${ }^{2}$, Bhanu Pratap Singh ${ }^{3}$
}

\author{
${ }^{1}$ MAA Institute of Speech and Hearing, Hyderabad, India \\ ${ }^{2}$ Ali Yavar Jung National institute of Speech and Hearing Disabilities, Divyangjan, Mumbai, India \\ ${ }^{3}$ Hind Institute of Medical Sciences, Sitapur, Uttar Pradesh, India
}

Received: 24 October 2020

Revised: 10 May 2021

Accepted: 11 May 2021

\author{
*Correspondence: \\ Dr. Udit Saxena, \\ E-mail: dr.uditsaxena@gmail.com
}

Copyright: ( ) the author(s), publisher and licensee Medip Academy. This is an open-access article distributed under the terms of the Creative Commons Attribution Non-Commercial License, which permits unrestricted non-commercial use, distribution, and reproduction in any medium, provided the original work is properly cited.

\begin{abstract}
Estimation of the middle ear functioning is necessary for the assessment and management of the middle ear disorders. It is also crucial for deciding upon the management of hearing loss. Though very important for audiologists and otologist clinically, there are many misconceptions behind the scientific principle/rationale and confusions related to the terminologies and units used for the measurements of middle ear functioning such as acoustic impedance, acoustic admittance, middle ear reflectance and middle ear absorbance. Literature included in this review was searched from book chapters, instrument manuals, journal articles, monographs, scientific guidelines and standard specifications on the related topics. This review work threw light and eases understanding on the principles used by different instruments to estimate various measures of middle ear functioning. It will enhance understanding of the measurements and also of the relation between different measurements of middle ear functioning. Clinically, it will help professionals in analyzing results better considering the measurements variables (if present) and bring continuity in reporting the results of middle ear functioning.
\end{abstract}

Keywords: Impedance, Admittance, Reflex, Tympanometry, Reflectance, Absorbance

\section{INTRODUCTION}

In otology practice, assessment of middle ear functioning is helpful in detecting middle ear disorders like ear drum pathology, middle ear effusion, ossicular disarticulation, otitis media and otosclerosis, their respective severity and infection status. ${ }^{1-4}$ Audiologically, middle ear measurements are useful in ruling out conductive pathology which its important especial considering management of the hearing loss and also in cross-checking pure tone audiometry reports.,

Clinically, functioning of the middle ear is commonly monitored by estimating "Acoustic impedance and/or acoustic admittance" under the term "Tympanometry or immitance audiometry or acoustic immitance". "Immitance audiometry or acoustic immitance is a blend used by many authors to describe both, acoustic impedance and acoustic admittance. ${ }^{5-13}$ This blend often caused confusion across the professionals as one instrument can either measure acoustic impedance or acoustic admittance. Newer instruments mostly provide the measure of acoustic admittance while the instruments dated back to 80's and 90's used to give acoustic impedance. Some may even use the term "static compliance" in place of acoustic admittance. ${ }^{6,11-12}$ One of the reasons for all the confusion is the fact that these measures are still are not well understood to the professionals especially their respective underlie principles. This has also led to poor reporting of the middle ear functioning tests. Peer literature on acoustic impedance 
and acoustic admittance is limited to the results of acoustic impedance and acoustic admittance tests but not their scientific basis, mathematical calculations and formulae involved. Technical information, whatever available, on acoustic impedance and acoustic admittance is scattered which further makes it difficult to understand these measures. Recent measures of middle ear functioning "Middle ear reflectance and middle ear absorbance" are largely unknown scientifically to a number of professionals globally. In depth understand of the measures of middle ear functioning is very important for the audiologists and otologist as it will help in making precise diagnosis and management decision. Hence, in this systematic review we aimed to collectively describe and organize principles, terminologies and units of the measures of middle ear functioning such as acoustic impedance, acoustic admittance, middle ear reflectance and middle ear absorbance in one document.

\section{METHODS}

For the purpose of this review article, authors had collected information from renowned book chapters, instrument manuals, indexed journal articles, monographs, scientific guidelines and standard specifications on related topics of the measures of middle ear functioning. Internet search of databases like scopus, pubmed, eric, sciencedirect and JASTOR was done. Different combinations of keywords; acoustic, impedance, admittance, static compliance, middle ear functioning and acoustic reflex, middle ear reflectance and middle ear absorbance were used as search strategy.

\section{Acoustic impedance, acoustic admittance, middle ear reflectance and middle ear absorbance}

Acoustic immittance: Acoustic impedance and acoustic admittance

Acoustic immittance is the term collectively used for acoustic impedance and acoustic admittance. ${ }^{14-16}$ Acoustic impedance refers to the resistance to the flow of sound energy through an acoustic medium while acoustic admittance describes the ease with which the sound energy is transmitted through an acoustic medium. ${ }^{13}$ The two measures are related reciprocally. ${ }^{7,13,15-17}$

\section{Principles of Acoustic impedance and acoustic admittance}

\section{Acoustic impedance}

Term "impedance" is first used by Heaviside (1886) to characterize a physical system in terms of power transfer or power consumption. ${ }^{15,} 17$ Conceptually, impedance comes from the Newton's second law that relates force (cause) and change in state (effect)..$^{7,13,15-17}$ Depending on the system type, impedance can be electrical, mechanical or acoustic. Electrical impedance is defined as the ratio of voltage (cause) to current (effect) and mechanical impedance as the ratio of force (cause) and velocity (effect). ${ }^{7,13,15-17}$ In acoustics, the sound pressure (p) of a time varying sinusoid, pure-tone, is a quantity similar to mechanical force and the resulting volume velocity (U) is similar to mechanical velocity. ${ }^{7,13,15-16,18}$ Therefore, acoustic impedance $(Z)$ is the ratio of sound pressure and volume velocity $(\mathrm{U})$. Sound pressure and volume velocity of time varying sinusoid are calculated as (equation 1),

$$
Z=\frac{p}{U}
$$

Sound pressure and volume velocity of time varying sinusoid are calculated as (equation 2 and 3 respectively),

$$
\begin{gathered}
p(t)=p_{0} \sin (2 \pi f t+\varphi) \\
U(t)=U_{0} \sin (2 \pi f t)
\end{gathered}
$$

where $\mathrm{p}_{0}$ and $\mathrm{U}_{0}$ are peak sound pressure and peak volume velocity, respectively; $\mathrm{f}$ is the frequency $(\mathrm{Hz})$ of sinusoid, $\mathrm{t}$ is time (seconds) and $\varphi$ is the phase angle that describes the time lag between sound pressure and volume velocity.

Going by the definition, acoustic impedance can be determined if the following two quantities are known; (a) ratio of sound pressure to volume velocity (equation 4),

$$
\frac{P_{0}}{U_{0}}
$$

a constant in a linear system for all the sound pressure inputs and (b) phase angle (equation 5),

$$
\varphi_{Z}=\frac{t_{p, U}}{T}
$$

where $\mathrm{T}$ is the time period and $t_{\mathrm{p}, \mathrm{U}}$ is the time difference between the sound pressure and volume velocity waveforms at corresponding point (Figure 1).

In the example shown (Figure 1), sound pressure led volume velocity therefore $\varphi_{\mathrm{z}}$ is positive. In condition where sound pressure lags volume velocity, $\varphi_{z}$ is negative.

\section{Acoustic admittance}

Acoustic admittance (Y) is the direct reciprocal of acoustic impedance (equation 6),

$$
Y=\frac{1}{Z}
$$

Therefore by equation 1 , equation 7 is as follows,

$$
Y=\frac{U}{p}
$$


and by equation 5 , phase angle for acoustic admittance is given by equation 8 ,

$$
\varphi_{Y}=\frac{360^{\circ} t_{U, p}}{T}
$$

here $t_{, p, U}$ is the time difference between volume velocity with that of sound pressure. ${ }^{7,13,15,16}$

Volume velocity lags sound pressure in the example shown (Figure 1), therefore, $\mathrm{t}_{\mathrm{U}, \mathrm{p}}=-\mathrm{t}_{\mathrm{p}, \mathrm{U}}$ and $\varphi_{\mathrm{Y}}=-\varphi_{\mathrm{Z}}$.

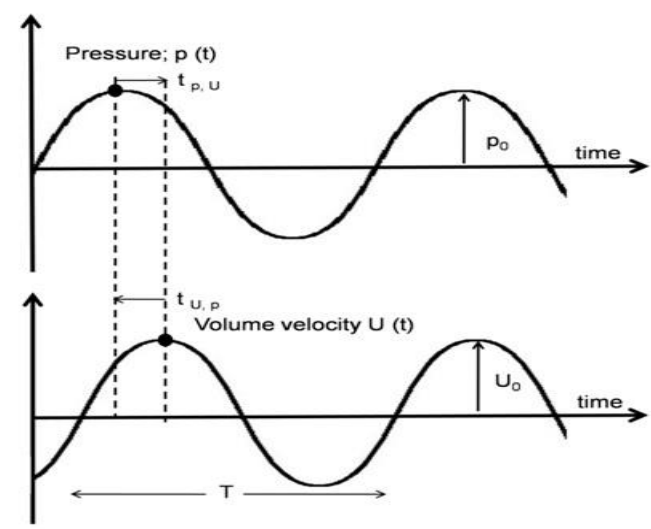

Figure 1: Time varying sinusoidal sound pressure (p) of time period $(\mathrm{T})$ and the resulting volume velocity

(U).

\section{Phasor representation of acoustic impedance and acoustic admittance}

Phasor diagram of acoustic impedance and acoustic admittance is given in Figure 2.,13,15,16 In these phasor diagrams, the length of the phasor is equivalent to the magnitude of acoustic impedance and acoustic admittance and angle between the horizontal axis and phasor represent the phase angle between sound pressure and volume velocity (Figure 2).

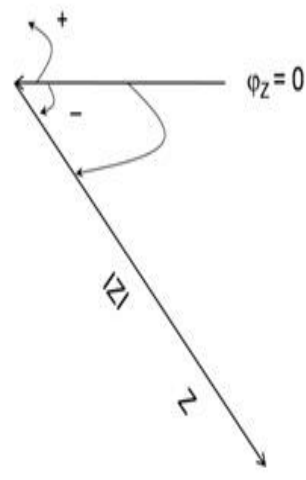

(A)

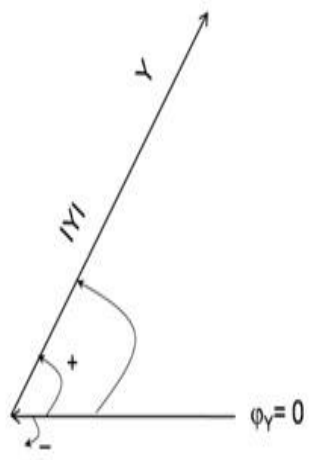

(B)
Figure 2: Phasor diagram of acoustic impedance (A) and acoustic admittance $(B)$.
Components of acoustic impedance and acoustic admittance

Both, acoustic impedance and acoustic admittance, have real and imaginary components. Real components of acoustic impedance and acoustic admittance are resistance (R) and conductance $(G)$, respectively. ${ }^{7,13,15,16}$ Imaginary components of acoustic impedance and acoustic admittance are reactance (X) and susceptance (B), respectively.,13,15,16 Rectangular notation of acoustic impedance and acoustic admittance with their respective imaginary and real components is shown in Figure 3.

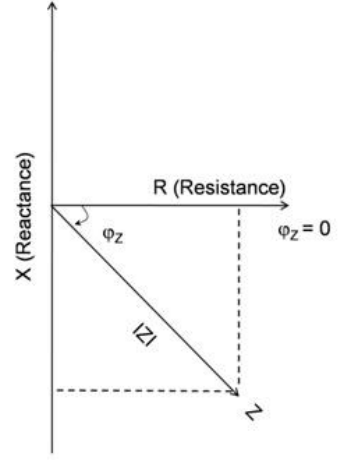

$|Z|=\sqrt{ } R^{2}+X^{2}$

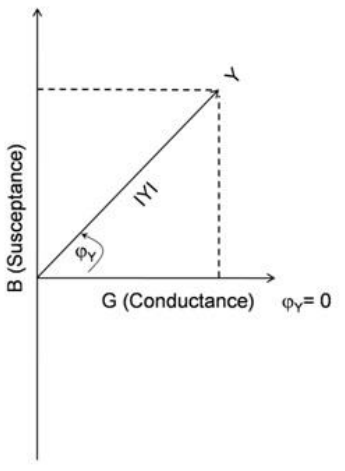

$|Y|=\sqrt{ } G^{2}+B^{2}$
Figure 3: Rectangular notation of acoustic impedance $(Z)$ and acoustic admittance with their real and imaginary components.

\section{Elements contributing to the acoustic impedance and acoustic admittance of middle ear}

A mechanical system has three elements (mass, spring and friction) that contribute to its overall impedance. Likewise, middle ear, an acoustic-mechanical system, is comprised of mass, spring and friction elements contributing to its acoustic impedance or acoustic admittance. ${ }^{7,13,15,16}$ Mass, spring and friction elements of middle ear are discussed in following sub-sections.

Mass elements

Mass elements of the middle ear system include tympanic membrane's pars flaccida, ossicles and perilymph fluid within cochlea. ${ }^{15}$ Acoustic impedance is directly proportional to mass (M) (equation 9),

$$
Z=\frac{p}{U} \propto \mathrm{M}
$$

whereas acoustic admittance is indirectly proportional to mass (equation 10). $7,13,15,16$

$$
Y=\frac{U}{p} \propto \frac{1}{M}
$$

By Van Camp \& Creten, equation 11 and 12 as, 


$$
\begin{aligned}
& |Z|=\frac{2 \pi f t M}{S^{2}} \\
& |Y|=2 \pi f t C S^{2}
\end{aligned}
$$

where $S$ is the surface area on which the sound pressure acts.

Rectangular components of acoustic impedance and acoustic admittance for mass element are given as equation 13 and 14 respectively,

$$
\begin{aligned}
& X=\frac{2 \pi f t M}{S^{2}}, R=0 \\
& B=\frac{S^{2}}{2 \pi f t M}, G=0
\end{aligned}
$$

Spring element

Elements of the middle ear system that acts like springs are ligaments, tendons, tympanic membrane and air enclosed in the ear canal and middle ear space. For spring element as equation 15 and 16 respectively 7,13,15,16

$$
\begin{aligned}
& Z=\frac{p}{U} \propto \mathrm{K} \propto \frac{1}{C} \\
& Y=\frac{U}{p} \propto \frac{1}{K} \propto \mathrm{C}
\end{aligned}
$$

where $\mathrm{K}$ is the spring constant and $\mathrm{C}$ is compliance which is reciprocal of the spring (equation 17).

$$
C=\frac{1}{K}
$$

By Van Camp \& Creten, equation 18 and 19 as,

$$
\begin{aligned}
& |Z|=\frac{1}{2 \pi \mathrm{ftCS}^{2}} \\
& |\mathrm{Y}|=2 \pi \mathrm{ftCS}{ }^{2}
\end{aligned}
$$

Rectangular components of acoustic impedance and acoustic admittance for spring element are given as equation 20 and 21.

$$
\begin{array}{r}
X=\frac{2 \pi f t M}{S^{2}}, R=0 \\
\mathrm{~B}=2 \pi \mathrm{ftCS} \text { and } \mathrm{G}=0
\end{array}
$$

Friction component
Mucous lining of middle ear cavity, narrow passages between middle ear cavity and mastoid contributes to the overall acoustic friction by the middle ear. ${ }^{15}$ For friction element as equation 22 and 23 respectively,

$$
\begin{aligned}
& |Z|=\frac{b}{S^{2}} \\
& |Y|=\frac{S^{2}}{b}
\end{aligned}
$$

where $b$ is the friction coefficient.

Rectangular components of acoustic impedance and acoustic admittance for friction element are given as equation 24 and 25 respectively,

$$
\begin{aligned}
& R=\frac{b}{S^{2}}, X=0 \\
& R=\frac{b}{S^{2}}, B=0
\end{aligned}
$$

Acoustic impedance and acoustic admittance of closed volume

Acoustic impedance and acoustic admittance of closed volume, in the present context "ear", is calculated as equation 26 and 27.

$$
\begin{aligned}
& |Z|=\frac{\rho c^{2}}{2 \pi f V} \\
& |Y|=\frac{2 \pi f V}{\rho c^{2}}
\end{aligned}
$$

Where $\rho$ is the density of air, $c$ is the speed of sound and $\mathrm{V}$ is volume.

On simplifying, equation 26 and 27, we get,

$$
\begin{aligned}
& |Z|=\frac{226059}{\mathrm{fV}} \\
& |Y|=\frac{f V}{226059}
\end{aligned}
$$

If volume is $1 \mathrm{~cm}^{3}$ (or $\mathrm{ml}$ ) and frequency is $226 \mathrm{~Hz},|\mathrm{Z}|=$ $1000 \mathrm{ohms}$ and $|\mathrm{Y}|=1 / 1000 \mathrm{ohms}$ or $1 \mathrm{mmho}$.

Hence is the notation "A volume of $1 \mathrm{~cm}^{3}$ (or $\mathrm{ml}$ ) has an acoustic impedance of $1000 \mathrm{ohms}$ and an acoustic admittance of $1 \mathrm{mmho}$ at standard atmospheric pressure at $226 \mathrm{~Hz}{ }^{15}$

\section{Instrument measuring acoustic impedance and acoustic admittance}

Instruments measuring acoustic impedance or acoustic admittance of the middle ear consist of a probe that comprises a small loudspeaker, an air pressure regulatory 


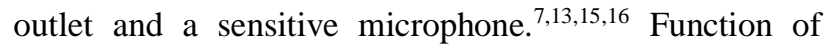
small loudspeaker is to present acoustic signal into the ear canal. A part of energy of the acoustic signal presented is absorbed by the middle ear and the rest is reflected back from the tympanic membrane Sound pressure level of which is then measured by the microphone. This sound pressure level is then processed by the instrument to provide acoustic impedance or acoustic admittance. ${ }^{7,13,15,16}$ Acoustic impedance or acoustic admittance is measured at different air pressure levels. It is the air pressure outlet that regulates air pressure in the ear canal. This is done particularly to monitor eustachian tube functioning and to determine ear canal volume.

Acoustic impedance meter

Working principle and measurement: It is known that,

$$
Z=\frac{p}{U}
$$

So, if the instrument maintains a constant volume velocity in the ear canal, then acoustic impedance is directly related to the voltage, microphone voltage or probe voltage $\left(\mathrm{V}_{\mathrm{m}}\right)$, measured at the microphone of the probe. ${ }^{7,13,15-17}$ Probe is considered to be a constant volume velocity source if $\mathrm{V}_{\mathrm{m}}$ and sound pressure level is linear for a range of known acoustic impedances. This linearity is ensured by measuring $\mathrm{V}_{\mathrm{m}}$ of calibrated volume cavities and if $\mathrm{V}_{\mathrm{m}}$ holds constant across the range of volume then the probe is considered to have a constant volume velocity source. Figure 4 shows an example of a probe that generates constant volume velocity for a range of volumes ( 0.5 to 2 $\mathrm{ml})$.

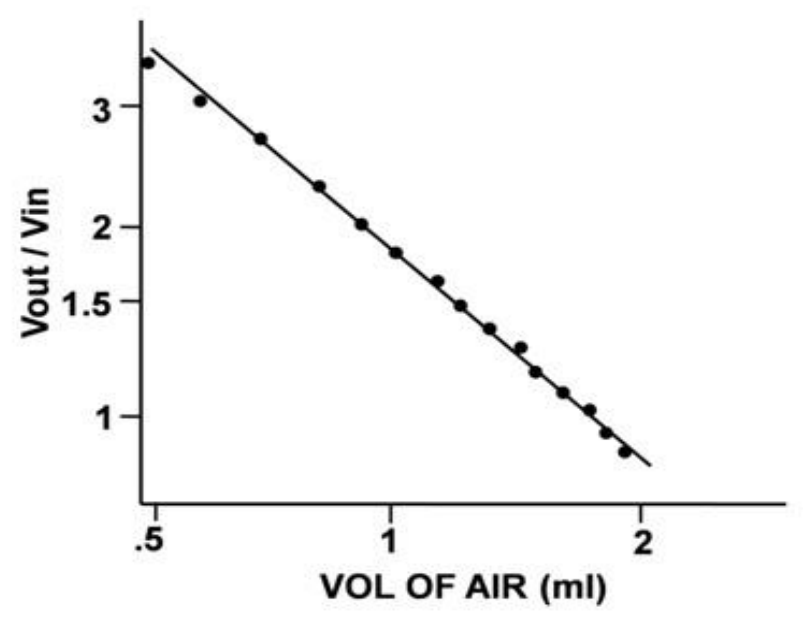

Figure 4: Example of a probe that generates constant volume velocity for a range of volume.

Acoustic signal at a certain sound pressure level is presented into the ear canal using a probe which is a constant volume velocity source. The voltage of this signal is usually specified as the reference voltage $\left(\mathrm{V}_{\text {ref }}\right)$. This acoustic signal after being reflected from tympanic membrane is received up by the microphone and its sound pressure level is measured and converted to relative voltage $\left(\mathrm{V}_{\mathrm{m}}\right)$. The microprocessor unit of the instrument then compares $V_{\text {ref }}$ and $V_{m}$ to give acoustic impedance value of the middle ear based on acoustic impedance measurements from already known acoustic impedances (These conversion values are manufacture specific and are not published).

Acoustic admittance meter (Instrument design and operation)

Working principle and measurement: It is known that,

$$
Y=\frac{U}{p}
$$

So, if the instrument retains a constant sound pressure level (p) in the ear canal then its acoustic admittance is directly related to the volume velocity. ${ }^{7,} 13,15-17$ The volume velocity is directly proportional to the voltage of the driver $\left(V_{d}\right.$, that generates it. ${ }^{15}$ Acoustic signal at specific sound pressure level and driver voltage $\left(\mathrm{V}_{\mathrm{d}}\right)$ is presented into the ear canal. This acoustic signal after being reflected from the tympanic membrane is received by the microphone, its sound pressure level is measured and converted into relative voltage $\left(\mathrm{V}_{\mathrm{m}}\right)$. In order to maintain a constant sound pressure level, acoustic admittance meter uses an automatic gain control circuit that adjusts the gain of the driver based on the voltage measured by the microphone $\left(\mathrm{V}_{\mathrm{m}}\right)$. The gain that is required to maintain a constant sound pressure level (p) is converted into the required acoustic admittance values based on the acoustic admittance of already known compliances values.

Acoustic impedance and acoustic admittance measurement at the level of tympanic membrane..$^{7,13,15-17}$

Measurement done using probe in the ear canal gives acoustic impedance or acoustic admittance values of the combined, external ear and middle ear. Clinically required is the acoustic impedance or acoustic admittance of middle ear, not external ear. Thus, acoustic impedance or acoustic admittance of the external ear must be excluded. Air enclosed between the probe and tympanic membrane contributes to the acoustic impedance or acoustic admittance of the external ear. Ear (external ear canal and middle ear) is assumed to be composed of acoustically parallel circuits because sound pressure that acts at the level of probe and at the level of tympanic membrane is same especially for low frequencies. Overall acoustic impedance or acoustic admittance of the combined elements in parallel circuits (external ear canal and middle ear) are given as equation 28 and 29 respectively as,

$$
\begin{aligned}
& \frac{1}{Z_{o}}=\frac{1}{Z_{c}}+\frac{1}{Z_{m}} \\
& Y_{o}=Y_{c}+Y_{m}
\end{aligned}
$$


where o, c and m represent overall, external ear canal and middle ear, respectively.

Tympanic membrane is considered to be like a rigid wall at extreme positive $(+400 \mathrm{daPa})$ and an extreme negative (-200 daPa) air pressure (Regulated by air pressure outlet). At these air pressures, acoustic impedance or acoustic admittance measured by the probe is essentially of the ear canal. Therefore, when overall measurement and measurement at extreme air pressures are available, acoustic impedance or acoustic admittance of the middle ear can be calculated using equation 27 and 28 . Measurements done at $+400 \mathrm{daPa}$ and $-200 \mathrm{daPa}$ also provide ear canal volume.

\section{Measurement units of the components of immittance audiometer}

C.G.S unit of acoustic impedance and acoustic admittance is ohm and mho, respectively. Acoustic impedance or acoustic admittance can also be expressed in terms of volume $(\mathrm{ml} / \mathrm{cc})$ under standard atmospheric condition or if corrected for change in these-condition., ${ }^{2,19,20}$ Some instruments have also used the term static compliance ( $\mathrm{ml}$ or cc) for acoustic impedance and acoustic admittance. ${ }^{2,19,20}$

\section{Acoustic reflex measurement}

Acoustic reflex measurements are helpful in the detection of conductive, cochlear and retro-cochlear disorders., ${ }^{71-22}$ Acoustic reflex estimation depends on the measurement of acoustic impedance or acoustic admittance. ${ }^{23}$ Instrument that measure acoustic reflex monitor acoustic impedance or acoustic admittance while the reflex activator is presented. Acoustic reflex is said to be activated when acoustic impedance increase or acoustic admittance decrease by $0.02 \mathrm{ohm} / \mathrm{mmho}$ or $0.03 \mathrm{ohm} / \mathrm{mmho}$ in presence of reflex activator. ${ }^{21-24}$

\section{Multifrequency-multicomponent tympanometry}

Multifrequency-multicomponent tympanometry was designed to provide elaborated view of the middle ear functioning when compared to single-frequencycomponent acoustic admittance., 8 Multifrequencymulticomponent tympanometry involves estimation of the multiple components of acoustic admittance, including conductance, susceptance and phase angle (all these components are described in the previous sections), at more than one frequency ranging from $226 \mathrm{~Hz}$ to $2000 \mathrm{~Hz}$ rather than measurement of only "acoustic admittance" at one frequency "usually $226 \mathrm{~Hz}$ ". 7, 8

\section{Middle ear reflectance and middle ear absorbance}

For a long period of time, audiologists and otologists, remained dependent on single frequency or narrow range frequency measures such as impedance increase, acoustic admittance and multifrequency-multicomponent tympanometry for estimating middle ear function. However, these measures have limitation of not providing information about the acoustic characteristics of normal middle ear or changes in the acoustic characteristics of middle ear due to any disorder. Middle ear reflectance (MER) and middle ear absorbance (MEA) are wideband measurement of the middle ear function that provide information about how much sound energy is reflected and absorbed respectively, by the middle ear over a wide range of frequency. Middle ear reflectance is defined as the ratio of sound energy reflected at the tympanic membrane to sound energy incident to the middle ear. ${ }^{25}$ Middle ear absorbance is a simple modification of middle ear reflectance. ${ }^{26}$ Previously, middle ear absorbance was also called "transmittance" by Keefe and Simmons (2003). ${ }^{27}$ Considering, the energy conservation law and that no sound energy is absorbed by the ear canal walls the two measurements are related as (equation 30);

$$
\mathrm{MEA}=1-\mathrm{MER}
$$

Liu et al suggested that clinically the interpretation of the middle ear function based on the middle ear absorbance estimation would be rather easy than middle ear reflectance. ${ }^{26}$

\section{Measurement principle}

Wideband clicks are used as a stimulus in middle ear reflectance/middle ear absorbance measurements. These clicks are specifically developed to have approximately flat spectrum from 226 to $8000 \mathrm{~Hz}$. Clicks are delivered into the ear canal using probe receiver particular sound pressure levels. Some part of the sound energy in the clicks is absorbed by the middle ear and the other part gets reflected back from the tympanic membrane. The sound energy reflected back from the tympanic membrane gets picked up the probe microphone and undergoes Fourier transformation.

Following Fourier transformation of the reflected sound energy into discrete frequency bins, sound pressure of specific frequency is calculated and compared with the sound pressure of the same frequency in the incident signal to calculate middle ear reflectance/middle ear absorbance. Calculation of middle ear reflectance and middle ear absorbance are as follows:

(a) Pressure reflectance $[\mathrm{Rp}(\mathrm{f})]$ at a particular frequency (f) is calculated using the equation mentioned below (equation 31):

$$
R p(f)=\frac{P r(f)-Q(f)}{R_{0}(f) P(f)+Q(f)}
$$

Where $\operatorname{Pr}(\mathrm{f})$ is the mean of reflected sound pressure at frequency (f), Q(f) is the incident sound pressure and $R_{o}$ is the source reflectance. 
(b) Middle ear reflectance is calculated by squaring the pressure reflectance (equation 32);

$$
\operatorname{MER}=\operatorname{IRp}(f) I^{2}
$$

(c) Middle ear absorbance: by equation 30 and 32,

$$
\operatorname{MEA}=1-\operatorname{IRp}(f) I^{2} \text { (equation 32) }
$$

\section{Measurement units of middle ear reflectance and middle ear absorbance}

Middle ear reflectance and middle ear absorbance are dimensionless quantity as it is calculated from the ratio of non-dimensionless quantity i.e., sound pressures of incident and reflected sound energy. They are either represented as number ( 0 to 1$)$ or in percentage ( 0 to $100 \%)$. For example, at specific frequency 1 or $100 \%$ middle ear reflectance means that tympanic membrane reflected back entire sound energy while 0 or $0 \%$ middle ear reflectance indicate that middle ear absorbed all sound energy incident at the tympanic membrane.

Conversely, 1 or $100 \%$ middle ear absorbance indicate that all the sound energy at that frequency is absorbed by the middle ear while 0 or $0 \%$ middle ear absorbance means middle ear did not absorb anything.

Commercially, TITAN is the only clinically available instrument available to measure middle ear absorbance. ${ }^{28}$ It provides middle ear absorbance estimation for 107 specific frequencies from $226 \mathrm{~Hz}$ to $8000 \mathrm{~Hz}$ and by varying the pressure between +200 to $-400 \mathrm{daPa}$. Figure 5 shows example of middle ear absorbance measured at 0 daPa.

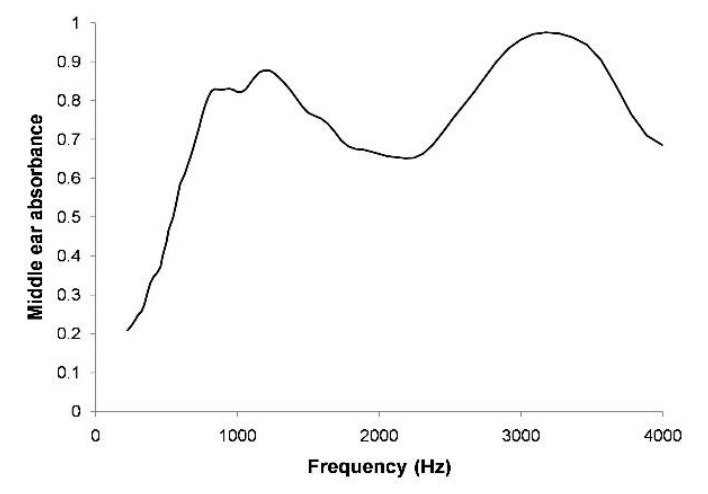

Figure 5: Example of middle ear absorbance measured at 0 daPa.

\section{DISCUSSION}

Measurements of the middle ear functioning are very important in the field of Otology and Audiology. Over a period of 4 decade a lot of technological advancement took place in the instrument developed for the purpose of measuring middle ear functions. There was a gap in the literature where all the measures and their respective concepts were not explained and compared in the same document. ${ }^{1-12}$ That has complicated the learning of the various middle ear measures especially in the trainee students. For the critical analysis of any test's result, it is important to know the physical principles/rationale behind the measure and the instrument used for estimating that measure. Nowhere in literature the physical concepts and principle of middle ear measures are explained thoroughly and again in one document. ${ }^{1-12}$ One of the reasons for that being older literature just focused on the traditional measurements of middle ear measure (acoustic impedance, acoustic admittance, acoustic reflex and multicomponent/multicomponent tympanometry) and new literature on latest measures (middle ear reflectance and middle ear absorbance). But in the end, all the measures are of middle ear functions and understanding of one enhance the understanding of other. Further, it is necessary to know why, how and the course of the development of the various tests of middle ear functions. ${ }^{1-28}$ This review work has overcome that issue.

Most of the scientific concepts related to acoustic impedance, acoustic admittance, acoustic reflex and multicomponent/multicomponent tympanometry are same. They are sound pressure, volume velocity, peak sound pressure, peak volume velocity, resistance, reactance, phase angle, time difference between sound pressure and volume velocity, frequency, time period, ,mass, surface area on which the sound pressure acts, spring constant, compliance, friction coefficient, density of air, speed of sound, volume, microphone voltage or probe voltage, reference voltage and driver voltage. ${ }^{7,13,15-17}$ Different ones are reactance and resistance which are specific to acoustic impedance \& susceptance and conductance and susceptance to acoustic admittance..$^{7,13,15-17}$ Pressure reflectance, reflected sound pressure, incident sound pressure and source reflectance are the concepts to be understood in the measurement of middle ear reflectance and middle ear absorbance. ${ }^{25-28}$ All these concepts along with their formulae and units are covered comprehensively in this review.

\section{CONCLUSION}

In this review we have provided detailed information on the principles behind the measurements of acoustic impedance, acoustic admittance, acoustic reflex, multicomponent/multicomponent tympanometry, middle ear reflectance and middle ear absorbance along with the terminologies and units used for them. Clinically, this review will help audiologists and otologists in analyzing the result of middle ear functioning better considering the measurements variables (if present) and will bring continuity in reporting the results of middle ear functioning as well. Further, this will help audiologists and otologists in selecting one measure over the other 
depending on the requirement of information-type. Normative data on acoustic impedance, acoustic admittance, middle ear reflectance and middle ear absorbance is not reviewed. Future review may incorporate them.

Funding: No funding sources

Conflict of interest: None declared

Ethical approval: Not required

\section{REFERENCES}

1. Dhingra PL, Dhingra S. Diseases of ear, nose and throat. 7th ed. India: Elsevier; 2017.

2. Hunter LL, Shahnaz N. Acoustic immittance measures: Basic and advanced practice. 1st ed. San Diego, USA: Plural Publishing; 2013.

3. Silman S, Silverman CA, Arick DS. Acousticimmittance screening for detection of middle-ear effusion in children. J Am Acad Audiol. 1992;3(4):262-8.

4. Stach B. The audiologists assessment tools: Immitance measures. Clinical audiology: An introduction. Delmar, USA: Cengage Learning; 2008: 314-354.

5. ANSI. American national standards specification for instruments to measure aural acoustic impedance and admittance (aural acoustic immittance). New York, USA: American National Standards Institute; 1987.

6. American Speech-Language-Hearing Association. Guidelines for screening for hearing impairment and middle-ear disorders. ASHA. 1990:(2):25-30.

7. Fowler CG, Shanks JE. Tympanometry. In: Katz J, Burkard RF, Medwetsky L, eds. Handbook of clinical audiology. 5th ed. Philedelphia, USA: Lippincott Williams \& Wilkins: 2002.

8. Hunter LL, Sanford CA. Tympanometry and wideband acoustic immitance. In: Katz J, Chasin M, English K, Hood LJ, Terry KM, eds. Handbook of clinical audiology. 7th ed. Philedelphia, USA: Lippincott Williams \& Wilkins; 2015: 137-163.

9. Gelfand SA. Acoustic immittance assessment. In: Gelfand SA, eds. Essentials of audiology. 3rd ed. New York, USA: Thieme; 2011: 205-238.

10. Martin FN, Clark JG. Diagnostic Hearing Tests. In: Martin FN, and Clark JG, eds. Introduction to audiology. 9th ed. Boston, USA: Allyn and bacon; 2006: 148-185.

11. Shanks JE, Lilly DJ, Margolis RH, Wiley TL, Wilson RH. Tympanometry. J Speech Hear Disord. 1988;53(4):354-77.

12. Wiley TL, Fowler CG. Acoustic immittance measures in clinical audiology: A primer. San Diego, USA: Singular Publishing; 1997.

13. Wiley TL, Stoppenbach DT. Basic principles of acoustic immittance measures. In: Katz J, Burkard RF, and Medwetsky L, eds. Handbook of clinical audiology. 5th ed. Philedelphia, USA: Lippincott Williams \& Wilkins; 2002.

14. Popelka GR. Acoustic immittance measures: terminology and instrumentation. Ear Hear. 1984;5(5):262-7.

15. Camp KJ, Margolis RH, Wilson RH, Creten WL, Shanks JE. Principles of tympanometry. ASHA Monogr. 1986;(24):1-88.

16. Camp KJ, Creten WL. Principles of acoustic impedance and admittance. In: Felman AS, Wilber A, eds. Acoustic impedance and admittance-the measurement of middle ear function. Baltimore, USA: Williams \& Wilkins; 1976: 300-335.

17. Margolis RH, Hunter LL. Tympanometry: Basic principles and clinical applications. In: Musiek FE, Rintelmann WI, eds. Contemporary perspectives in hearing assessment. Boston, USA: Allyn \& Bacon; 1999: 89-130.

18. Bernstein JL. Audio systems, 1966. Available at: https://en.wikipedia.org/wiki/JL_Audio. Accessed on 18 October 2020.

19. Gelfand SA. Essentials of audiology. 2nd ed. New York, USA: Thieme; 2001.

20. Northern JL. Acoustic Immittance Measures in Clinical Audiology: A Primer. Ear Hear. 1998;19(2):168.

21. Saxena U, Allan C, Allen P. Crossed and uncrossed acoustic reflex growth functions in normal-hearing adults, typically developing children, and children with suspected auditory processing disorder. Int $\mathbf{J}$ Audiol. 2015;54(9):620-6.

22. Saxena U, Allan C, Allen P. Acoustic reflexes in normal-hearing adults, typically developing children, and children with suspected auditory processing disorder: Thresholds, real-ear corrections, and the role of static compliance on estimates. J Am Acad Audiol. 2017;28(6):480-90.

23. Bennett M. Impedance Concepts Relating to the Acoustic Reflex. The acoustic reflex: Basic principles and clinical applications. Florida, USA: Academic Press; 1984: 36-59.

24. Schairer KS, Feeney MP, Sanford CA. Acoustic Reflex Measurement. Ear Hear. 2013;34:43-7.

25. Keefe DH, Levi E. Maturation of the middle and external ears: acoustic power-based responses and reflectance tympanometry. Ear Hear. 1996;17(5): 361-73.

26. Liu YW, Sanford CA, Ellison JC, Fitzpatrick DF, Gorga MP, Keefe DH. Wideband absorbance tympanometry using pressure sweeps: System development and results on adults with normal hearing. J Acoust Soc Am. 2008;124(6):3708-19.

27. Keefe DH, Simmons JL. Energy transmittance predicts conductive hearing loss in older children and adults. J Acoust Soc Am. 2003;114(6):3217-38.

28. Interacoustics. TITAN-Instruction for use. 2013.

Cite this article as: Saxena U, Kumar SBR, Singh BP. Measurements of middle ear functioningtraditional (acoustic impedance and acoustic admittance) and modern (middle ear reflectance and middle ear absorbance): a review Int $\mathrm{J}$ Otorhinolaryngol Head Neck Surg 2021;7:1068-75. 